Journal of Engineering and Applied Sciences 14 (9): 2808-2812, 2019

ISSN: 1816-949X

(C) Medwell Journals, 2019

\title{
Channel Variation Effects on Video Streaming of WiMAX Networks
}

\author{
Hussein M. Hathal \\ Department of Electrical Engineering, Faculty of Engineering, \\ Al-Mustansiriyah University, Baghdad, Iraq
}

\begin{abstract}
Video streaming stay one of internet services that became important services to subscribers. Based on WiMAX networks, this service will provide to all subscribers that used internet. WiMAX networks provide advantages for real time application for internet protocols such as video streaming or voice. In this study, variation effect of channel types evaluated on video streaming of WiMAX which are (vehicular, suburban, outdoor to indoor and free space). Scheduling services discussed depending on real time application service. OPNET Simulator Version 14.5 is used to model the design proposed. Best results are achieved for throughput using rtPS with suburban channel type and QAM code modulation. Minimum packet delay (end-to-end) achieved using ertPS with channel type (free space) and QAM code modulation.
\end{abstract}

\section{Key words: ertPS, UGS, QAM, rtPS, WiMAX, VoIP, IPTV, QoS, OPNET}

\section{INTRODUCTION}

All manu scripts must be in English. These guidelines include complete description soft the fonts, spacing and related information for producing your proceedings manu scripts. Please follow the mand if you have any questions, direct the MTO the production editor incharge of your proceedings.

"For reach the communication techniques based on high frequency (microwave), one of solutions to this is to use WiMAX technology. WiMAX technique is one of the best technologies that provide high data transfer rate with cover large areas and the loss rate of data relatively small" (Hathal et al., 2016a, b). The coverage area of WiMAX technique for fixed station is up to $50 \mathrm{~km}$ and for mobile station up to $15 \mathrm{~km}$ (Abdulhussein, 2016).

Five types of service in WiMAX are based on IEEE Stander 802.16 that is UGS, ertPS, rtPS, nrtPS and BE. Three types of service are used for real-time applications which rtPS, UGS and ertPS. Unsolicited Grant Service (UGS) can be used in real-time applications such as VOIP and streaming video, it is non-favorite in use due to data loss (Abdulhussein, 2016). Second service type is named real time Polling Service (rtPS). This service type is able to transfer packets with variable data rates in real time, such as VOIP and video streaming. In every polling interval, the Base station (Bs) doing a poll for users in base station's cell when know the user that required to connect with it, carry out a (bandwidth request). Then, base station grant to burst data using UL-MAP to sure receiving of data (Hamodi et al., 2013). Third service type is named extended real time PS (ertPS). It is suitable for applications that need to cancel silence during the internet protocol communication such as video streaming and voice (Hathal et al., 2016a).
"Video information available from providers of video service as the content of the video. Content of video structured as a series of video frames or images that are transmitted or received of shared and present in the context of a fixed rate. Depending on stream of video in real-time and temporary storage requirements from the server and the network, the contents of the video are classified by several parts including of video format, pixel color depth, encoding type and the frame rate interval (Hamodi and Thool, 2013; Gorbenko et al., 2015).

International Telecommunication Union in addition to the International Standards Organization have been developed types of technologies related compression techniques. The most important feature of this type of modulation is to reduce the required contents of the bandwidth for achieved encoding by reducing the distance between the contents of the frame. Recommended type named Advanced Video Codec (AVC) used to publish IPTV in future. They suggested that the best distribution which automatically adjusts the size of the frame to encode H.264 Video is the gamma distribution (Hamodi and Thool, 2013).

Literature review: Hathal et al. (2016a) discussed the Modulation Coding effect (MC) variation for Voice over Internet Protocol (VoIP) that effect on WiMAX networks. He is study the Quality of Service (QoS) performance of scheduling types such as ertPS, rtPS and UGS based on OPNET simulator. He found that the QPSK coding modulation with ( $1 / 2$ coding rate) has best enhancement compared with other code modulation types. 
Abdulhussein (2016) studied the variation effect of channel types on Voice over Internet Protocol (VoIP). He study variable channels with effects on performance of QoS parameters as Jitter, packet delay (end to end), Throughput and packet loss. He found that vehicular environment type of channel was significant results compared with other channel types.

Study of Hamodi et al. (2013), Internet Protocol Television (IPTV) performance was studied over fixed system of WiMAX with different coding modulation. They found that code modulation types (16 and 64 QAM) had better performance than QPSK.

Hathal et al. (2016a) discussed the effect of code modulation variation on Internet Protocol Television (IPTV) of WIMAX networks. They are study the QoS parameters that effect on different types of MC. They found that best results for decreasing delay was QAM code modulation with coding rate $3 / 4$.

Hamodi and Thool (2013) studied the modulation coding effect on streaming video over WIMAX. They are found that by using OPNET Simulator, video codec type Scalable Video Coding (SVC) for streaming video over WiMAX was an appropriate codec video if compared with Advanced Video Coding (AVC).

In study of AbdulHussein et al. (2016), a brief discussion done for modulation code variation in non-real time application of WiMAX networks. They are found that QPSK modulation code type with $1 / 2$ coding rate has best results if it compared with the other modulation code types.

Hamodi et al. (2014) studied variable keys effect on (IPTV) for WIMAX networks. The keys that studied were variation channel, variation modulation coding and variation speed of mobile user. Results indicates that coding modulation type called (adaptive modulation) can offer best results of QoS.

In study of Alreshoodi et al. (2016), Quality of Experience (QoE) was studied and compared with QoS. They are used fuzzy interface to estimate Quality of Experience $(\mathrm{QoE})$. They are found that $\mathrm{QoE}$ present saving in power and bandwidth more than QoS.

\section{MATERIALS AND METHODS}

Channel modulation types: A briefly discussion will be explained about channel types. Virtual modeling, based on OPNET Simulator, supposed that different types of channel to evaluation the performance of proposed system. In this study, 4 types of channel modeling was used which is (vehicular, suburban, out door to in door and free space). These types is as follow in Table 1 (Hamodi et al., 2013, 2014).

Video architecture and streaming in WiMAX: A brief description of the architecture of IPTV over WiMAX will done in this study. In order to ensure the quality of TV broadcasts across the internet networks, service must be suitable for the network. Architecture of IPTV is divided into four sections. The first section is the contents of video. The contents of video is the first sub system that storing any type of video with audio. In the second section, the contents of video converts to the encoded data. Third section sends it over long distances using WiMAX. While the fourth section will distribute the content of video. Where it is offer a high capacity in the content distribution through the WiMAX network (Hamodi and Thool, 2013). As shown in Fig. 1,

Table 1: Channel types

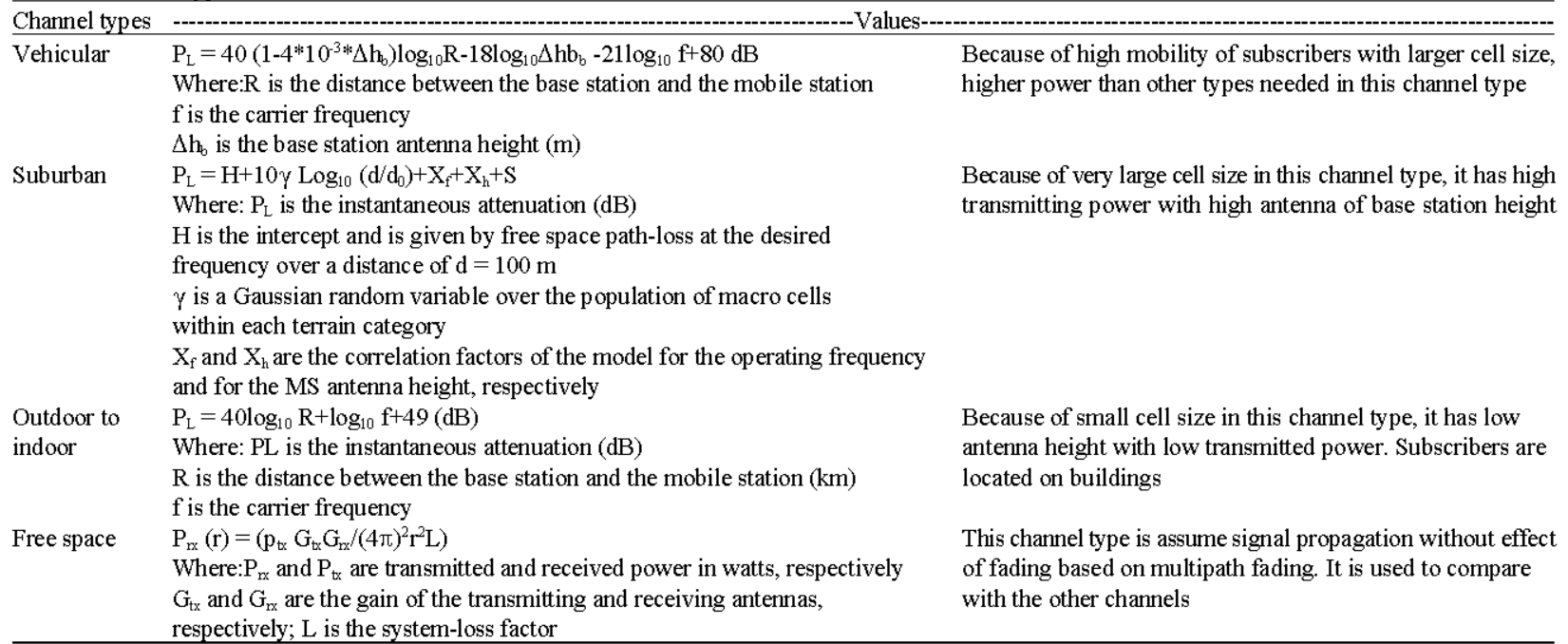




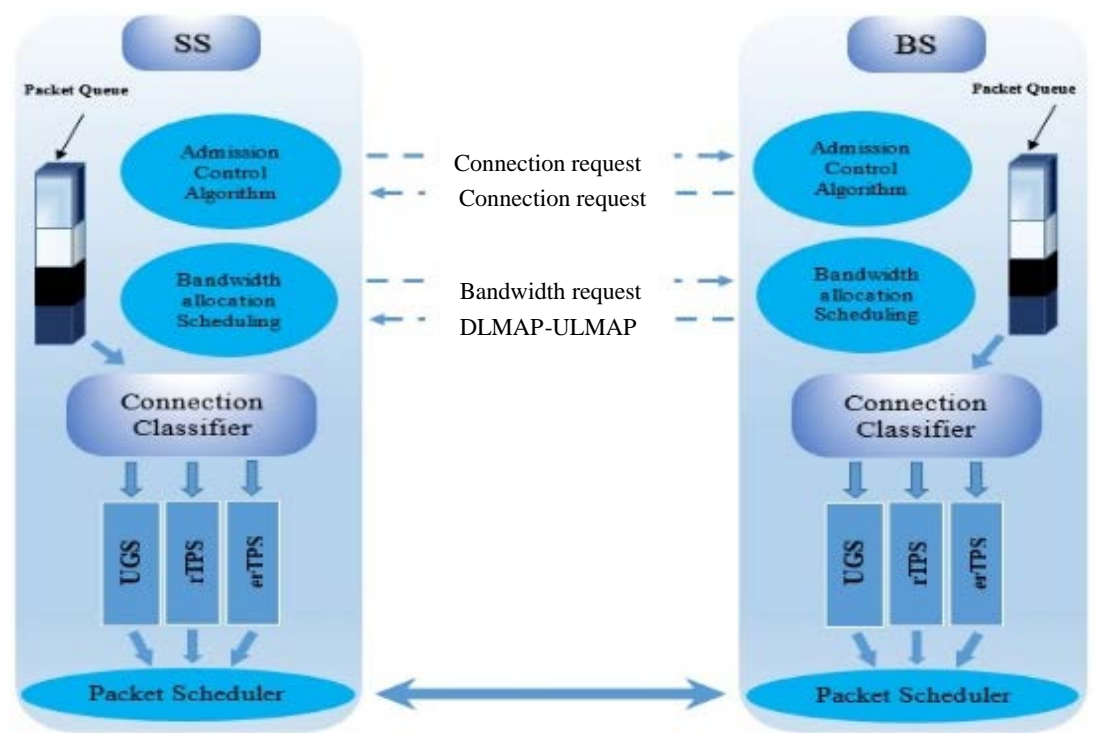

Fig. 1: Video streaming architecture in WiMAX system

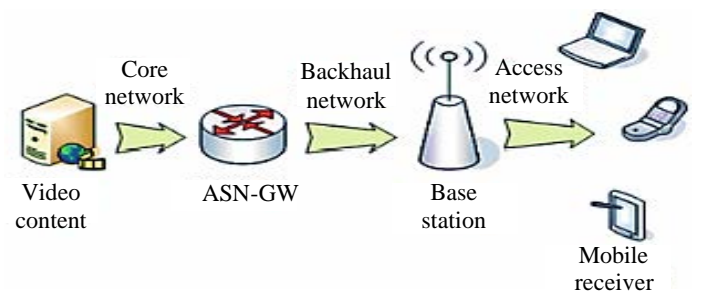

Fig. 2: QoS mechanism at MAC level

(Hamodi et al., 2014). Depending on survey of deploying video streaming, there are two video traffic requirements as illustrated.

Quality of Experience (QoE): QoE is measured by the user's knowledge of the service. Mean Opinion Score (MOS) traditionally measured QoE for voice and video that based on the knowledge of the quality of sending and receiving voice and picture depending on sample population. Real-time services quality for video streaming measured based on the International Telecommunication Union and Telecommunication Standardization Sector (ITU-T) for circuit switched networks (Hamodi and Thool, 2013).

Quality of Services (QoS): In order to satisfy the client request, Subscribers (SS) are needs bandwidth allocation. There are several types of QoS which is make the same idea with different quality. Figure 2 shows QoS mechanism in packet networks on the WiMAX BS and SS is illustrated as Gorbenko et al. (2015).

QoS tested parameters: Four parameters will be tested for this design, these parameters is illustrated as follows:
Jitter: Simply is the difference in time between two packets transmitted from node 1 and received at node 2 . Mathematical representation of this example is illustrated as follow in Eq. 1:

$$
\text { Jitter }=(\mathrm{T} 4-\mathrm{T} 3)-(\mathrm{T} 2-\mathrm{T} 1)
$$

Where:

$\mathrm{T} 1$ and $\mathrm{T} 2=$ Transmitted packets

$\mathrm{T} 3$ and $\mathrm{T} 4=$ Received packets

Sometimes negative jitter occurs because of the difference in receiving time was less than the difference of the transmitting time (Hamodi and Thool, 2013).

End to end delay: is defined as all the delay occur from the beginning of video streaming from node 1 , through the channel, to video received at node 2. Mathematical representation of this parameter is illustrated as follows Eq. 2:

$$
\mathrm{De} 2 \mathrm{e}=\mathrm{Dn}+\mathrm{De}+\mathrm{Dd}+\mathrm{Dc}+\mathrm{Dde}
$$

where Dn, De, Dd, Dc and Dde represent the network, encoding, decoding, compression and decompression delay, respectively (Hathal et al., 2016a; Abdulhussein, 2016).

Packet drop: is defined as inability of one or more packets to reach the receiver node. It is occur because of scrambled network. Mathematical representation of this parameter is as follow in Eq. 3:

$$
\mathrm{Pl}=\text { Lost packet/(Lost_packet-Received packet) }
$$




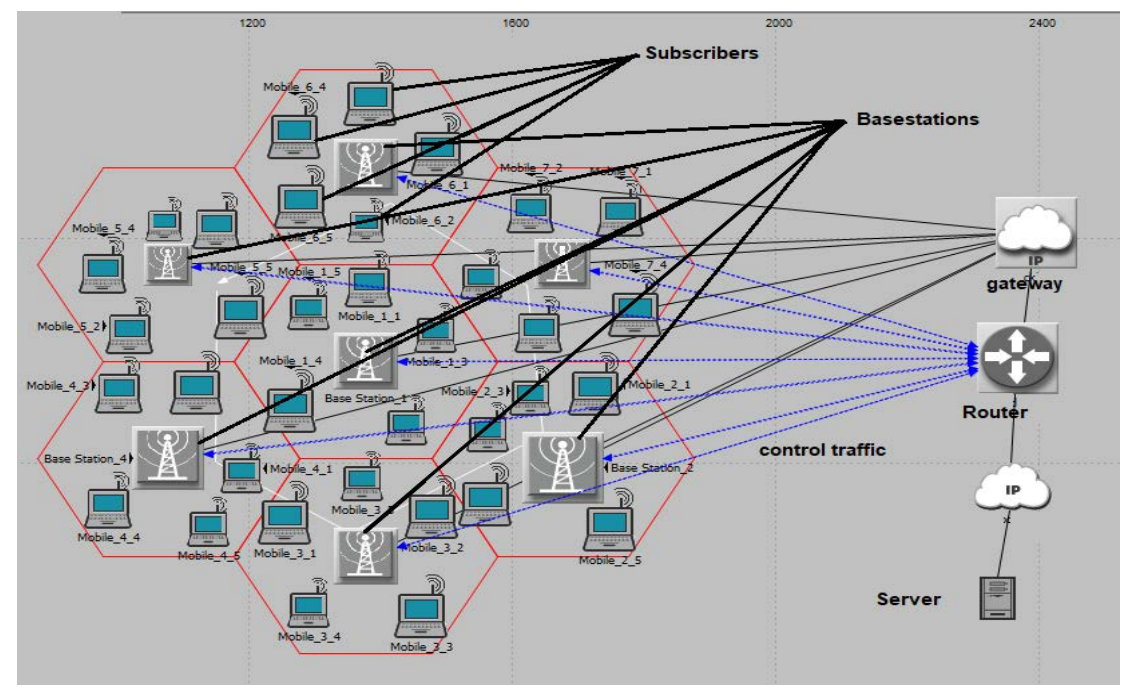

Fig. 3: Model design of simulated system

Table 2: Parameters of simulated system

\begin{tabular}{ll}
\hline System elements & Values \\
Antenna gain & $15 \mathrm{dBi}$ \\
System design shape & Hexagonal \\
No. of cells & 7 \\
No. of subscriber/cell & 5 \\
Speed of mobile subscriber & $50 \mathrm{~km} / \mathrm{h}$ \\
Traffic characteristic & Streaming video \\
Maximum transmitted power (W) & 0.5 \\
Physical profile type & OFDM \\
Physical profile frequency & $20 \mathrm{MHz}$ \\
\hline
\end{tabular}

Throughput: Is defined as a function of complexity and associated video content. The units of throughput is bits/second. Throughput traffic loading has dynamic nature because variable bit rate is loaded from channel to network. Peak range of throughput is achieved from variable rate load (AL-Hawawreh and Zreikat, 2017).

System simulation: "The scenario of system design is shown in Fig. 3. Proposed system contains 7 hexagonal cells. Every cell contain one base station with 5 mobile subscribers. Three scheduling services types (rtPS, UGS and ertPS) that are used to check the best of proposed system for QoS parameters. Channel variation applied on this system to evaluate the results of video streaming in WiMAX. Depending on the results of Hathal et al. (2016a) that found the best code modulation of the system was QAM, this type of code modulation is used in the system. OPNET Simulator Version 14.5 used to model the system. QoS parameters that tested was (Jitter, packet end-to-end delay, data drop and throughput). The parameters of system design is illustrated in Table 2.

\section{RESULTS AND DISCUSSION}

Video jitter case results are shown in Fig. 4. These results are found, for all channels of system, Jitter in

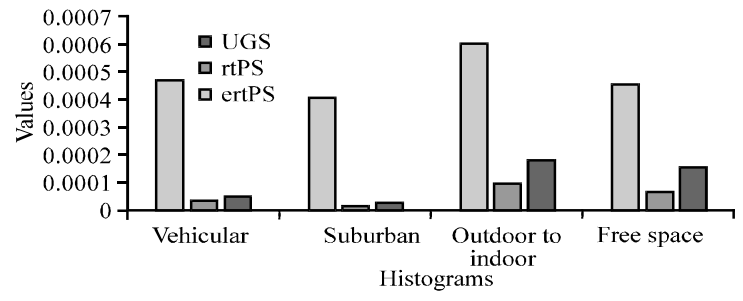

Fig. 4: Video Jitter histograms (sec); Jitter (sec)

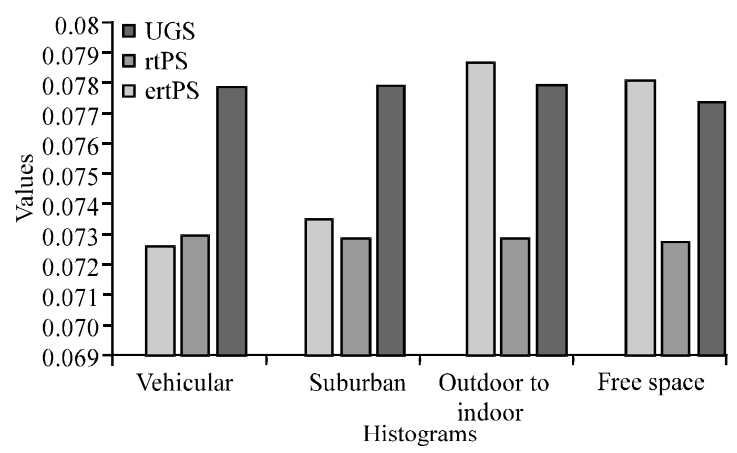

Fig. 5: Packet end-to-end delay histograms (sec); Packet end-to-end delay ( $\mathrm{sec}$ )

ertPS scheduling services has maximum values compared with other scheduling services (rtPS and UGS). These results are different of Hamodi et al. (2014). The rtPS of Hamodi et al. (2014) out performance of proposed system. These differences in results are done because Hamodi et al. (2014) does not depend on QAM modulation coding as a reference code.

Packet end-to-end delay results are shown in Fig. 5. Because of large fading occurred in outdoor to indoor channel, maximum delay is occurred in ertPS 


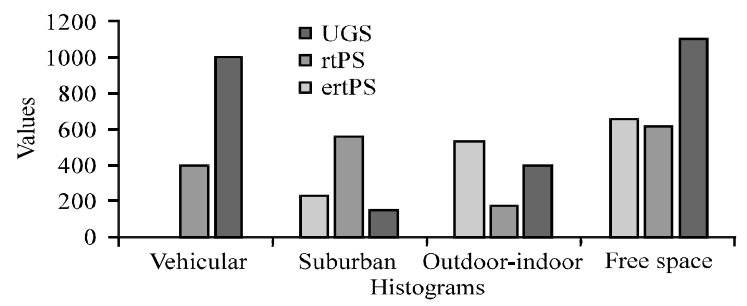

Fig. 6: Packet drop histograms (bits/sec); Base station 1 WiMax data dropped (bits/sec)

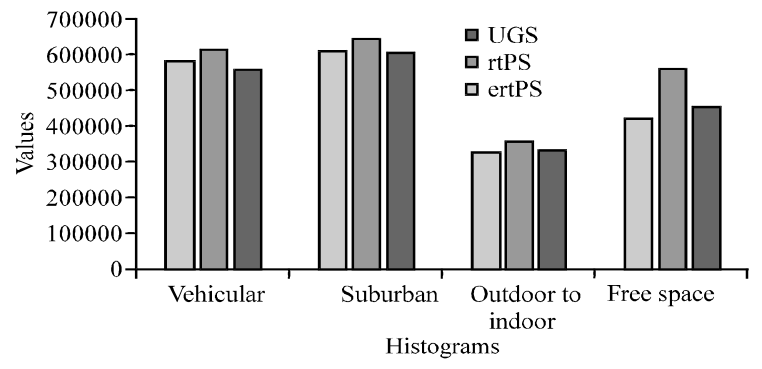

Fig. 7: Video throughput histograms (bits/sec); Average (WiMax throughput (bits/sec))

scheduling services. Minimum results are found in rtPS scheduling services for all channels. Better results for rtPS scheduling services is found compared with Hamodi et al. (2014) in free space channel type. These differences is occurred because of no fading occur in free space channel type.

Data drop results are shown in Fig. 6. These results show that maximum data drop occurred in free space for UGS scheduling services. Minimum results done in vehicular channel for ertPS.

Throughput results are shown in Fig. 7. These results found that maximum throughput is done using suburban channel for scheduling services rtPS type. Different results are found if it compared with Hamodi et al. (2014). For free space channel Hamodi et al. (2014) explain that minimum throughput occurred in this channel type but minimum throughput results in proposed system are occurred in the outdoor-to-indoor channel type. These differences achieved because of no fading in free space and large fading found in outdoor-to-indoor channel type.

\section{CONCLUSION}

In this study, video streaming for WiMAX networks is treated with different channel types such as (vehicular, suburban, outdoor-to-indoor and free space) and with different scheduling services (rtPS, UGS, ertPS). It is evaluate performance of QoS as (jitter, packet end-to-end delay, data drop and throughput) in the proposed system. It is found that the suburban channel type has good results for video throughput in scheduling services rtPS type. For packet delay (end-to-end), it is found that minimum results found in free space. This result was better than Hamodi et al. (2014). Jitter of rtPS scheduling services was minimum in results for all channel types compared with Hamodi et al. (2014), these results of jitter were better than Hamodi et al. (2014). The conclusion of this study for these results is illustrated as follows.

For maximum throughput applications, QAM code modulation with suburban channel and rtPS scheduling services is suitable to give best results. For minimum packet delay (end-to-end), QAM code modulation with ertPS scheduling services and free space is suitable to give best results.

\section{REFERENCES}

AL-Hawawreh, M.S. and A.I. Zreikat, 2017. Performance analysis of a wimax network in different propagation models. Intl. J. Comput. Sci. Inf. Sec., 15: 603-609.

Abdulhussein, S.A., 2016. Performance evaluation of variable channels for WiMAX network. Intl. J. Res. Comput. Commun. Technol., 5: 425-428.

Alreshoodi, M., E. Danish, J. Woods, A. Fernando and F. Alarfaj, 2016. QoE-enabled efficient resource allocation for H. 264 video streaming over WiMAX. Proceedings of the IEEE International Conference on Consumer Electronics (ICCE), January 7-11, 2016, IEEE, Las Vegas, Nevada, USA., ISBN:978-1-46738363-9, pp: 251-252.

Gorbenko, N., E. Jean-Pierre, W. Almuhtadi and A. Srinivasan, 2015. Comparison of simulated and real network traffic results for multimedia streaming over WiMAX networks with QoS scheduling. Proceedings of the IEEE 28th Canadian Conference on Electrical and Computer Engineering (CCECE), May 3-6, 2015, IEEE, Halifax, Canada, ISBN:978-1-4799-5827-6, pp: 1324-1328.

Hamodi, J., K. Salah and R. Thool, 2013. Evaluating the performance of IPTV over fixed WiMAX. Intl. J. Comput. Appl., 84: 1-9.

Hamodi, J., R. Thool, K. Salah, A. Alsagaf and Y. Holba, 2014. Performance study of mobile TV over mobile WiMAX considering different modulation and coding techniques. Intl. J. Commun. Network Syst. Sci., 7: 10-21.

Hamodi, J.M. and R.C. Thool, 2013. Investigate the performance evaluation of IPTV over WiMAX networks. Intl. J. Comput. Networks Commun., 5:81-95.

Hathal, H.M., K.I. Sarmad and A.A. Riyadh, 2016. Performance evaluation of different modulation coding for non real time scheduling services in WIMAX Networks. Saussurea J., 6: 183-189.

Hathal, H.M., K.I. Sarmad and R.A. Abdulhussein, 2016. Performance evaluation of different modulation coding for real time scheduling services in video streaming of WiMAX networks. Saussurea J.,6: 27-33. 